\title{
Modulation of Oscillatory Power and Connectivity in the Human Posterior Cingulate Cortex Supports the Encoding and Retrieval of Episodic Memories
}

\author{
Bradley Lega' ${ }^{1}$, James Germi ${ }^{1}$, and Michael D. Rugg ${ }^{2}$
}

\begin{abstract}
Existing data from noninvasive studies have led researchers to posit that the posterior cingulate cortex (PCC) supports mnemonic processes: It exhibits degeneration in memory disorders, and fMRI investigations have demonstrated memoryrelated activation principally during the retrieval of memory items. Despite these data, the role of the PCC in episodic memory has received only limited treatment using the spatial and temporal precision of intracranial EEG, with previous analyses focused on item retrieval. Using data gathered from 21 human participants who underwent stereo-EEG for seizure localization, we characterized oscillatory patterns in the PCC during the encoding and retrieval of episodic memories. We identified a subsequent memory effect during item encoding characterized by increased gamma band oscillatory power and a low-frequency power desynchronization. Fourteen participants had stereotactic electrodes located simultaneously in the hippocampus and PCC,
\end{abstract}

\section{INTRODUCTION}

The role of the posterior cingulate cortex (PCC) in episodic memories has not been well characterized by intracranial EEG (iEEG). A pioneering study in this field identified an increase in high gamma band power during item retrieval (Foster, Dastjerdi, \& Parvizi, 2012); however, encoding-related effects have not been described in the literature, and the existing studies were conducted using surface electrodes placed in the interhemispheric fissure, which introduces signal from PCC-adjacent structures such as the precuneus (Foster, Rangarajan, Shirer, \& Parvizi, 2015; Foster, Kaveh, Dastjerdi, Miller, \& Parvizi, 2013; Watrous, Lee, et al., 2013). Moreover, there are no studies examining oscillatory properties of the PCC simultaneously with the ipsilateral hippocampus (rather than adjacent temporal lobe regions such as parahippocampus). This is despite considerable evidence gathered from fMRI studies that the PCC plays an important role in episodic memory retrieval and encoding (Vilberg \& Rugg, 2009; Cabeza, Ciaramelli, Olson, \& Moscovitch, 2008; Wagner, Shannon, Kahn, \& Buckner, 2005).

${ }^{1}$ University of Texas-Southwestern Medical Center, ${ }^{2}$ University of Texas at Dallas and with these unique data, we describe connectivity changes between these structures that predict successful item encoding and that precede item retrieval. Oscillatory power during retrieval matched the pattern we observed during encoding, with lowfrequency (below $15 \mathrm{~Hz}$ ) desynchronization and a gamma band (especially high gamma, 70-180 Hz) power increase. Encoding is characterized by synchrony between the hippocampus and PCC, centered at $3 \mathrm{~Hz}$, consistent with other observations of properties of this oscillation akin to those for rodent theta activity. We discuss our findings in light of existing theories of episodic memory processing, including the information via desynchronization hypothesis and retrieved context theory, and examine how our data fit with existing theories for the functional role of the PCC. These include a postulated role for the PCC in modulating internally directed attention and for representing or integrating contextual information for memory items.
Anatomically, the PCC is well situated to participate in episodic memory processes. Via the cingulum bundle, the mesial parietal area directly connects with both entorhinal cortex and pFC (De Schotten, Dell'Acqua, Valabregue, \& Catani, 2012), and white matter tractography studies imply that cingulum bundle degeneration here is selectively associated with memory deficits (Choo et al., 2010; Fellgiebel et al., 2005). These anatomical connections suggest that understanding oscillatory processes in the PCC during memory encoding may help explicate data for theta-frequency power changes observed in human hippocampal recordings (Jacobs, 2014), including the prevalence of $\sim 3-\mathrm{Hz}$ activity observed in episodic memory and spatial navigation (Lega, Jacobs, \& Kahana, 2011; Watrous, Fried, \& Ekstrom, 2011). The PCC participates in the default mode network (Greicius, Krasnow, Reiss, \& Menon, 2003), so examining PCC oscillatory activity during an episodic memory task may inform theories of how this network participates in mnemonic processing.

Successful episodic retrieval is associated with enhanced fMRI BOLD signal in the PCC, whereas successful encoding is associated with a relative reduction in BOLD activity (Spaniol et al., 2009; Maddock, Garrett, \& Buonocore, 2001). There is, however, no consensus theory describing 
the role of the PCC in episodic memory or indeed in cognition in general (Leech \& Sharp, 2014). It has been proposed that the region may provide a signal that shifts attention from internal to externally derived representations, may be part of a more general arousal mechanism, or may fill a more nuanced role in determining the scope of attentional focus during task execution (Elward \& Rugg, 2015; Cabeza et al., 2008; Wagner et al., 2005). Given the association of PCC degeneration with Alzheimer's disease and (limited) lesion evidence of cingulate involvement specifically in memory, the PCC may fulfill a more specific mnemonic role such as supporting the representation of contextual information during retrieval (Elward \& Rugg, 2015; Devinsky \& Luciano, 1993). Existing iEEG studies focusing on temporal-parietal connections have identified different connectivity patterns for the precuneus and lateral parietal cortex during successful item retrieval (Foster et al., 2013; Watrous, Lee, et al., 2013), and there is also evidence that, for recognition memory, gamma band activity in the lateral parietal cortex exhibits differences within the time series before participants render a novel/ old decision (Gonzalez et al., 2015). However, an examination of oscillatory activity during both memory encoding and retrieval specifically for the posterior cingulate has not been attempted, and these studies have not included direct hippocampal recordings.

We sought to directly examine PCC oscillatory activity in human adult patients with epilepsy. We collected data from 21 participants who underwent stereo-EEG (sEEG) for localization of seizure activity as part of a phase 2 implantation (Table 1). We examined within-site oscillatory activity during encoding and recall of single words, hypothesizing that we would observe a gamma band effect only during item retrieval based on fMRI data. This proved incorrect: We instead identified for the first time in the PCC oscillatory patterns during item encoding that predict successful memory formation. We also examined hippocampal-cingulate connectivity as quantified by phase synchrony. Our data demonstrated a pattern of 3-Hz connectivity that is decreased during successful item encoding but then increases before item retrieval. Synchrony both before and after study item presentation at this frequency predicted successful versus unsuccessful encoding. We analyzed oscillatory activity during item retrieval and identified a gamma band power increase commensurate with observed BOLD changes for this phase of memory. We place these observations in the context of existing theories of posterior cingulate function during episodic memory encoding and retrieval.

\section{METHODS}

We analyzed data from 21 participants who participated in a free recall task while implanted with sEEG electrodes precisely targeted to the PCC. Localization of all electrodes was performed by presurgical planning followed by stereotactic placement of depth electrodes and post- operative fusion of CT images with the preoperative MRI (Figure 1; Lega, Dionisio, Bingaman, Najm, \& GonzalezMartinez, 2014). Given the rarity of recordings in this brain region and the limited size of our data set, we elected not to separately analyze left versus right or PCC subregions. All electrodes underwent expert neuroradiology review to confirm placement as part of standard clinical practice. Patients were recruited from the epilepsy surgery practice at our medical center over a 2 -year period. All patients who had intracranial electrodes placed within the posterior cingulate and were able to participate in cognitive testing (complete one full session of the free recall task) were included in the analysis. The participants performed a free recall task to allow examination of neural activity during episodic memory encoding and retrieval. Testing sessions occurred only if the patient had been seizure free for 2 full hr before the session, and if a seizure occurred during the testing session, this session was not included in the analysis. They completed an average of 2.4 sessions, each consisting of the presentation of 20 fifteen-word study lists, followed by a period during which free recall of the words was attempted. Details of the behavioral task have been published (Sederberg, Kahana, Howard, Donner, \& Madsen, 2003). Items were considered correctly recalled if they were vocalized immediately after the list in which they were presented; if they occurred later in the task, they were considered previous list intrusions. Participants recalled an average of 3.8 words per study list (range $=1.35-$ 6.3 items).

In 14 of 21 participants, expert neuroradiology review confirmed that there was at least one contact in the hippocampus and one in the ipsilateral posterior cingulate gyrus. The total numbers of hippocampal and posterior cingulate contacts were 73 (38 left sided) and 62 (20 left sided), respectively. This resulted in 123 unique contact pairs between the hippocampus and posterior cingulate (67 left sided). There were no electrodes included from radiographically abnormal brain locations, including hippocampal sclerosis or possible neoplasia. Electrodes at sites of frequent interictal discharges were not included. Site of language dominance was determined from preimplantation clinical evaluation including neuropsychiatric assessments, fMRI, and Wada testing.

\section{Analysis of Power Differences}

We extracted oscillatory power and phase in each encoding trial in an 1800-msec window after item presentation using Morlet wavelets as previously described (Wave number 6; van Vugt, Sederberg, \& Kahana, 2007). We employed an automated artifact rejection algorithm to exclude noise and interictal spiking activity (kurtosis threshold of 4; Lega et al., 2014; Sederberg et al., 2007). Data were rereferenced using an average of all nonepileptic channels (those without seizure onset or interictal activity observed during clinical monitoring); 
Table 1. Description of Participants

\begin{tabular}{|c|c|c|c|c|c|c|c|c|}
\hline $\begin{array}{l}\text { Age at } \\
\text { Study }\end{array}$ & $\begin{array}{c}\text { Seizure } \\
\text { Duration } \\
\text { (years) }\end{array}$ & Seizure Onset Loc & $R P C C \#$ & $L P C C \#$ & R Ant Hipp \# & L Ant Hipp \# & $R$ Post Hipp \# & $L$ Post Hipp \# \\
\hline 62 & 15 & $\mathrm{R}$ ant insula & 2 & 0 & 0 & 3 & 2 & 0 \\
\hline 60 & 5 & L BA 38 & 0 & 1 & 0 & 4 & 0 & 4 \\
\hline 36 & 15 & R BA 7 & 3 & 0 & 0 & 0 & 0 & 0 \\
\hline 38 & 18 & R BAs 11-12 & 2 & 0 & 0 & 0 & 3 & 0 \\
\hline 30 & 4 & $\mathrm{R}$ post insula & 5 & 0 & 0 & 0 & 0 & 0 \\
\hline 17 & 5 & R BA 20 & 4 & 0 & 3 & 0 & 2 & 0 \\
\hline 54 & 23 & nonlocalizable & 2 & 0 & 0 & 0 & 0 & 0 \\
\hline 35 & 14 & R BA 21 & 2 & 2 & 2 & 2 & 2 & 2 \\
\hline 31 & 23 & R BA 39 & 3 & 0 & 0 & 0 & 2 & 0 \\
\hline 53 & 25 & R BA 19 & 2 & 0 & 0 & 0 & 2 & 0 \\
\hline 24 & 16 & L BAs 19-17 & 3 & 2 & 0 & 0 & 0 & 0 \\
\hline 20 & 5 & L hippocampus & 2 & 2 & 0 & 0 & 0 & 0 \\
\hline 18 & 11 & No seizures captured & 0 & 3 & 0 & 3 & 0 & 2 \\
\hline 62 & 16 & L BA 21, L BA 36 & 0 & 3 & 3 & 2 & 0 & 2 \\
\hline 26 & 24 & R BA 4 & 2 & 2 & 0 & 0 & 0 & 0 \\
\hline 32 & 16 & R BA 9 & 2 & 0 & 0 & 0 & 0 & 0 \\
\hline 39 & 10 & $\mathrm{R}$ hippocampus & 2 & 1 & 3 & 0 & 1 & 0 \\
\hline 30 & 4 & bilateral hippocampus & 0 & 2 & 2 & 3 & 0 & 1 \\
\hline 38 & 4 & R BA 36 & 2 & 0 & 0 & 0 & 0 & 0 \\
\hline 35 & 7 & $\mathrm{R}$ parahippocampus & 2 & 0 & 3 & 4 & 1 & 0 \\
\hline \multirow[t]{2}{*}{58} & 25 & no seizures captured & 2 & 2 & 3 & 3 & 1 & 1 \\
\hline & & Total & 42 & 20 & 19 & 24 & 16 & 14 \\
\hline
\end{tabular}

Number of sEEG electrode contacts within each region listed by participant. All electrodes included were not the site of seizure onset as determined by expert neurology review.

$\mathrm{BA}(\mathrm{s})=$ Brodmann's area(s); Seizure Onset Loc $=$ location of seizure onset as determined by sEEG; ant $=$ anterior; post $=$ posterior; Hipp $=$ hippocampus; $\mathrm{L}=$ left; $\mathrm{R}=$ right.

data were sampled at $1 \mathrm{kHz}$. However, in eight of the participants, clinical preference led to the application of a noise reduction filter during data acquisition on the commercial system, leading to loss of signal in the high gamma range above $180 \mathrm{~Hz}$. We therefore report the results of the power analysis up to a frequency of $180 \mathrm{~Hz}$, encompassing a wide swath of the high gamma range.

We looked for a subsequent memory effect during the encoding phase using a two-step method to combine across electrodes. First, we compared oscillatory power at $53 \log$-spaced frequencies centered at $\left.2^{(n / 8 ; n}=8: 68\right)$ between recalled and nonrecalled events via a (onetailed) rank sum test generating a $p$ value at each time-frequency pixel. We then shuffled the recalled/nonrecalled event labels 1000 times at each time-frequency point and identified the position of the true $p$ values within the distribution of $1000 p$ values from the test on shuffled data. True $p$ values at the extreme ends of this distribution indicated that oscillatory power was greater during either recalled or nonrecalled events. We used this to generate a $z$ value for time-frequency pixel for each electrode. Performing the statistical test within electrodes allowed us to combine data across electrodes using a test statistic, which avoids the need to normalize the power values, following previously published methods (Sederberg et al., 2003). Extreme positive or negative $z$ values represented strong positive or negative subsequent memory effects. In the second step of the analysis, we tested the resultant distribution of $z$ values (one value from each electrode at each time-frequency 
pixel) against zero; $z$ values significantly greater than zero indicated that oscillatory power was greater for recalled than nonrecalled events, and vice versa. We did this for electrodes in the hippocampi and PCC of each participant at each time-frequency pixel (Lega et al., 2014). We corrected for multiple comparisons in the time series data using the Benjamini and Hochberg false discovery rate (FDR) method with a $Q$ of 0.05 .

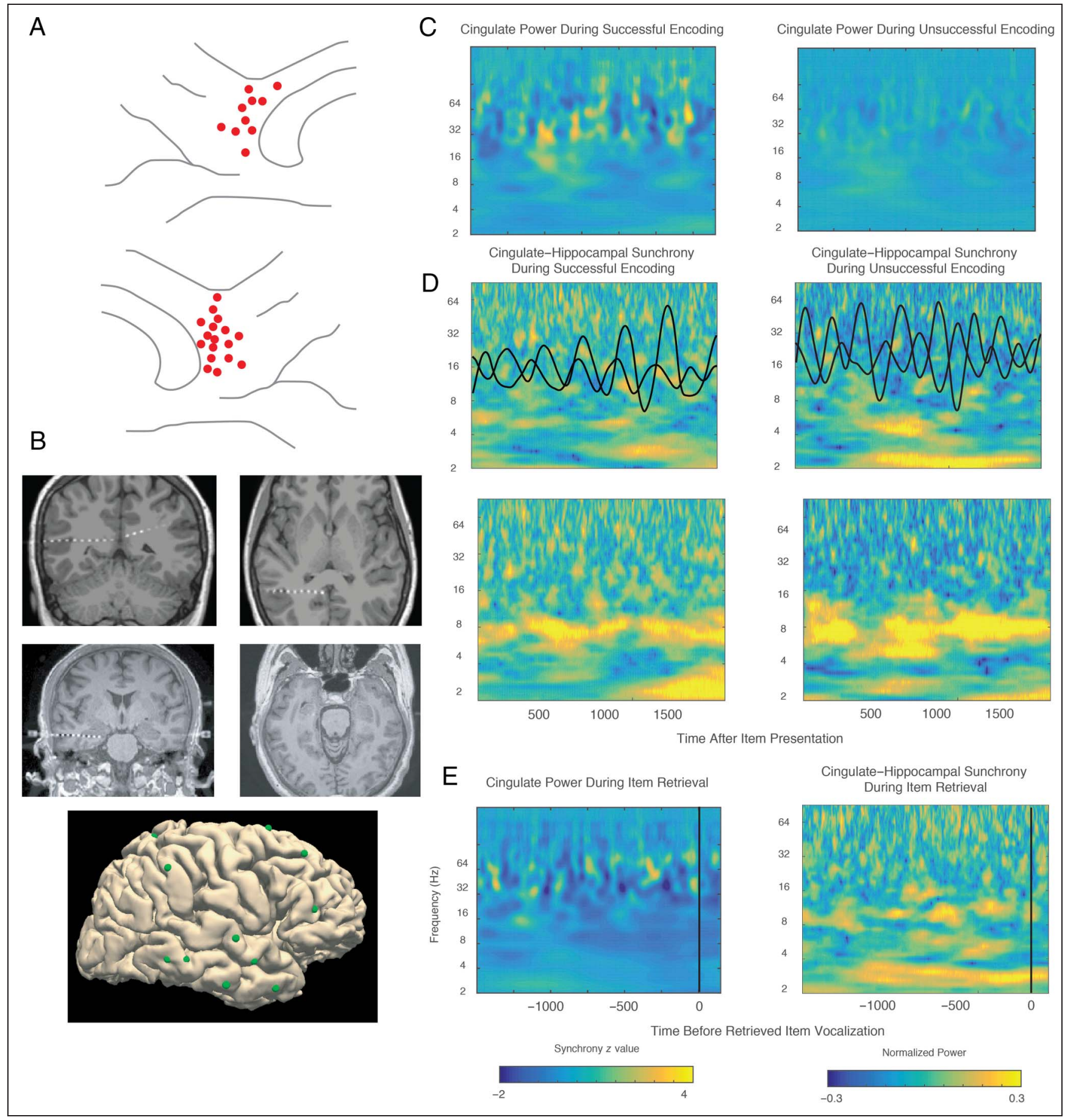

Figure 1. Description of electrodes in the data set. (A) Location of electrodes in the left and right posterior cingulate regions across 21 participants. Location was determined via expert neuroradiology review of electrode placement relative to the shown anatomical landmarks of the parieto-occipital sulcus, calcarine sulcus, cingulate sulcus, and corpus callosum following Kubota et al. (2013). (B) Example of contacts placed in the posterior cingulate and hippocampi with 3-D reconstruction showing insertion sites for sEEG electrodes. (C) Example of oscillatory power after presentation of recalled and nonrecalled items in a single electrode. (D) Examples of a single cingulate-hippocampal electrode pair exhibiting weak (left) and strong (right) 3- to 5-Hz synchrony during unsuccessful and successful encoding events, respectively. Superimposed is 3- to 5-Hz bandpassfiltered EEG from posterior cingulate and hippocampus for a single trial that exhibited stronger synchrony in nonrecalled condition. Two bottom panels of D show another electrode exhibiting synchrony more preferentially at $9 \mathrm{~Hz}$. (E) Oscillatory power in a single posterior cingulate electrode showing a sustained low-gamma power increase (left) and 3-Hz synchrony (right) before item retrieval. 
To further demonstrate the distribution of effects across participants, we compiled histograms of the number of electrodes exhibiting effects that exceeded the Type 1 error rate and then plotted time-frequency points at which this fraction was significantly greater than expected by chance using a binomial test. To compare the early versus late subsequent memory effect (SME) pattern in the posterior cingulate and hippocampus, we averaged the $z$ values across the early and later half of the time series and generated a matrix of $z$ value differences for each electrode. We then compared these distributions for hippocampal and cingulate electrodes using a $t$ test. We use the term "low frequency" to describe sub-beta oscillations, from 2 to $14 \mathrm{~Hz}$. We use the term "low gamma" to refer to the band from 40 to $70 \mathrm{~Hz}$ and "high gamma" to refer to the 70- to $250-\mathrm{Hz}$ frequency range. We use the term "slow theta" to describe oscillations in the 2.5- to 5-Hz range, although this range is more traditionally termed "delta." The issues surrounding the nomenclature and properties of this oscillation have been examined in several previous publications (Ekstrom \& Watrous, 2014; Jacobs, 2014).

\section{Analysis of Synchrony}

Using the wavelet-extracted power and phase information for the 14 participants with co-implanted hippocampal and PCC electrodes, we computed oscillatory synchrony between the hippocampal contacts and the posterior cingulate contacts. Calculation of oscillatory synchrony was performed with a Rayleigh test applied to the distribution of phase differences at each timefrequency pixel across all events, separately for recalled and nonrecalled events (Palva, Palva, \& Kaila, 2005). No baseline was removed from the wavelet-extracted power values for the analysis. The estimation of phase locking statistic (PLS) was then achieved with a shuffle procedure (200 shuffles) following Lachaux, Rodriguez, Martinerie, and Varela (1999). We randomly selected a subset of subsequently nonrecalled study items for the synchrony analysis to balance the number of successful and unsuccessful encoding trials while using the Rayleigh test. With this method, we extracted a single $z$ value per contact pair to quantify synchrony for different conditions (Lachaux et al., 1999). We tested for a memory encoding effect in hippocampal-PCC synchrony by applying a $t$ test with associated shuffle procedure to the distribution of $z$ values across all contact pairs (total $=$ 123) to determine a single test statistic at each timefrequency step. For both within-electrode power analyses and the electrode pair synchrony analyses, we used a shuffle procedure at each step comparing distributions between recalled and nonrecalled events. In general, we used parametric statistical tests ( $t$ test) when performing the test across test statistics (i.e., $z$ values) and nonparametric methods (rank sum test) when comparing distributions of measurements of signal (i.e., oscillatory power) directly.

\section{Retrieval Analysis}

For the retrieval phase, we employed a filtering procedure to identify word retrieval events that were separated from preceding or following retrieval events by at least 1500 msec (Burke et al., 2014). This resulted in an average of 81 retrieval events per participant. This number is lower than the number of successful encoding events because we filter only for retrieval events that are separated from other events by a large enough time window to analyze them independently (with a 1500 -msec window before and after). In free recall, the retrieval period is a 30-sec epoch after the math distractor task during which participants free recall as many memory items as they can from the list they have attempted to remember. Words that were not on any list they have seen are called extra list intrusions, whereas words from previous lists they have seen are called previous list intrusions (PLI); such retrieval events were excluded from the analysis. To analyze oscillatory power during the retrieval phase, we statistically compared power preceding vocalization of a retrieved memory item (period immediately preceding item vocalization) to average power drawn from $1500-\mathrm{msec}$ time windows randomly selected from throughout the retrieval phase. We matched this distribution of dummy events to the number of correct retrieval events that survived the filtering (described above), matching existing methods (Burke et al., 2014). We wanted to have a time window of sufficient length to analyze oscillations of less than $4 \mathrm{~Hz}$ given the previously observed importance of the slow theta frequency range for episodic processing. To examine synchrony, we computed $z$ values for hippocampalcingulate connectivity during the retrieval phase. We then compared the distribution of values at each point in the time series to synchrony values averaged from the same randomly selected 1500-msec time windows, as described above. This resulted in two matching distributions of $z$ values for each electrode pair. We used this to statistically test the distribution of synchrony $z$ values across all electrode pairs using a shuffle procedure as for the SME analysis.

\section{Analysis of Phase-Amplitude Coupling}

We quantified phase-amplitude coupling across the time series during encoding by binning high and low gamma power (10 phase bins) separately according to the phase of three frequency bands $(2.5-5,5-10$, and $11-14 \mathrm{~Hz})$ based on the observed differences in the SME analysis of power. We tested for nonuniformity across the binned power values using a circular ANOVA (Voytek, D'esposito, Crone, \& Knight, 2013). We extracted the $F$ statistic from the ANOVA and then derived a $z$ value using a shuffle procedure (shuffling the power time series, rebinning the power values, recalculating an $F$ statistic for 200 shuffles, and then deriving a $p$ value from this dummy distribution). This was done separately for recalled and nonrecalled events. We tested the resulting two distributions of test statistic values 
( $z$ values from the shuffle procedure) across electrodes using a $t$ test. We used circular regression to extract the preferred phase for coupling at each electrode for each band separately for recalled and nonrecalled events.

\section{RESULTS}

\section{Analysis of Power during Item Encoding}

We first analyzed oscillatory power separately in the hippocampus and posterior cingulate gyrus during memory encoding and retrieval. During item encoding, we timelocked the analysis to the presentation of the study items, conducting a standard subsequent memory analysis. All hippocampal electrodes included in the analysis were from participants who also had posterior cingulate electrodes. Analysis of oscillatory power is illustrated in Figures 2 and 3. Across 62 PCC contacts in 21 participants, there was significantly greater oscillatory power throughout the poststimulus epoch in the posterior cingulate during unsuccessful item encoding for low-frequency oscillations extending from 2.5 to $15 \mathrm{~Hz}(t(61)>2.99$ for significant

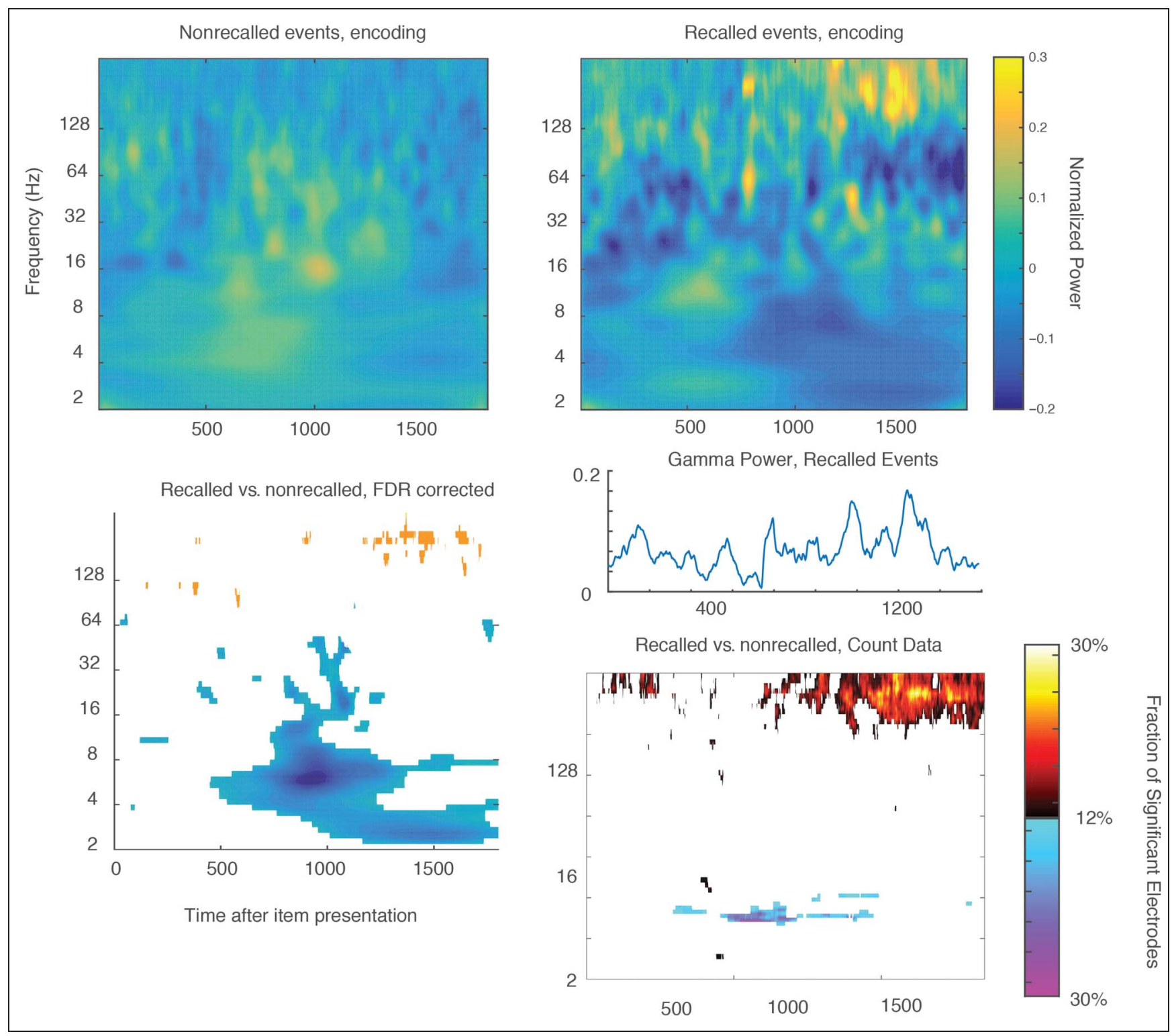

Figure 2. Power subsequent memory effect in the posterior cingulate. Top left plot shows average power during unsuccessful item encoding, whereas top right shows average power for successful encoding events across all participants. Statistical test was conducted initially between recalled and nonrecalled events within electrodes without normalization; normalized data are presented here to visualize effect across participants in the time series. Bottom left shows the difference in power for recalled versus nonrecalled events (the subsequent memory effect) for oscillatory power, with time-frequency steps that survive FDR correction with $Q=0.05$. Middle right shows high gamma oscillatory power across the time series, with a late increase $1300 \mathrm{msec}$ after item presentation. Bottom right plot shows a histogram of number of electrodes exhibiting a significant fraction of the total set of electrodes. Time-frequency pixels are plotted for which the significant number exceeds the fraction of electrodes expected by chance. Warm colors are pixels demonstrating recalled $>$ nonrecalled power, whereas cool colors show nonrecalled $>$ recalled. 


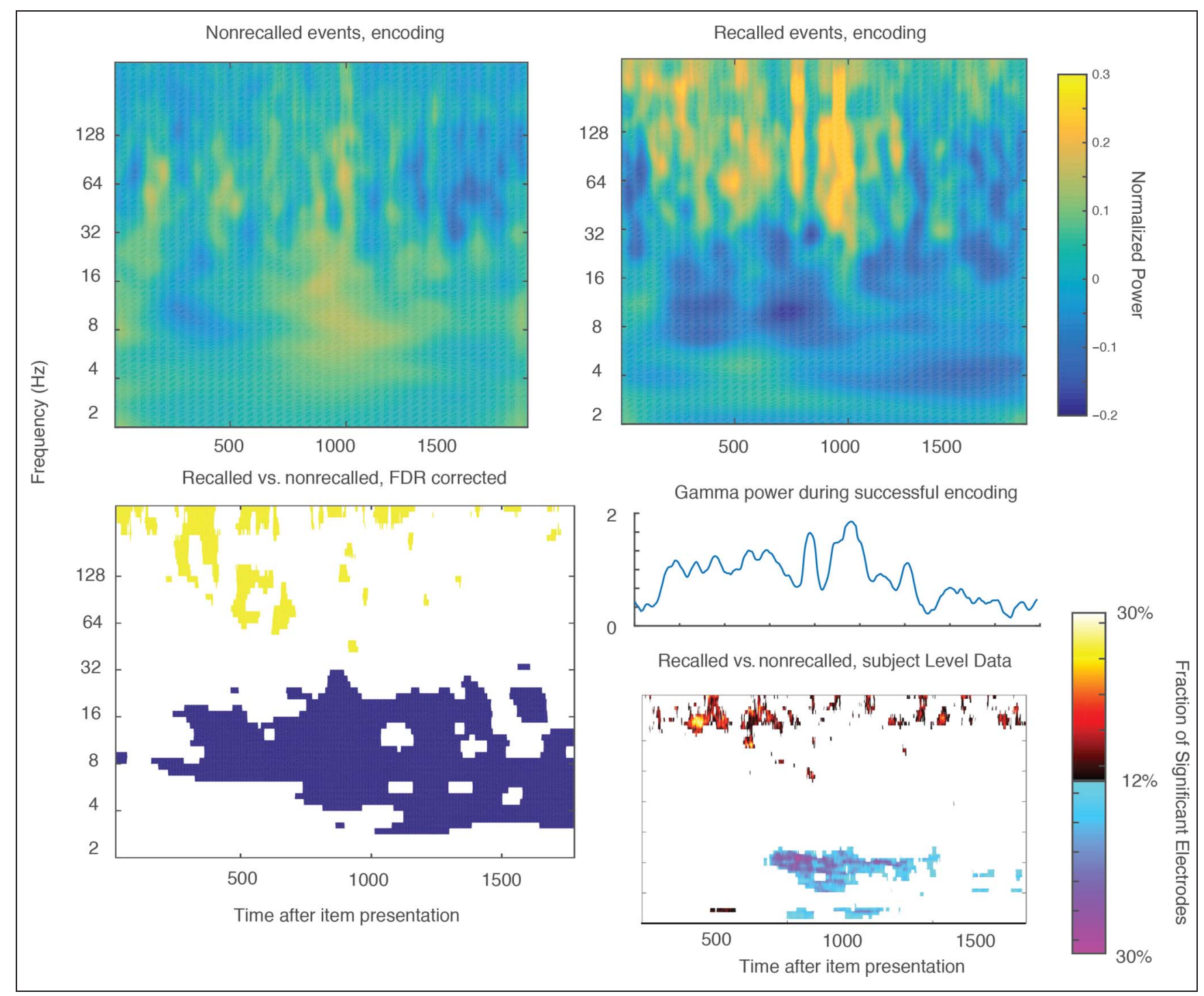

Figure 3. Power subsequent memory effect in the hippocampus. Hippocampal electrodes from 14 patients who also had posterior cingulate electrodes. Top left plot shows average power during unsuccessful item encoding, whereas top right shows average power for successful encoding events across all contacts in the data set. Bottom left shows the difference in power for recalled versus nonrecalled events (the subsequent memory effect) for oscillatory power, with time-frequency steps that survive FDR correction with $Q=0.05$. Middle right shows filtered gamma oscillatory power for recalled events, with a relative power increase in the first half of the time series. Bottom right shows time-frequency plot of histogram of count data, showing pixels for which the fraction of significant electrodes exceeded the Type 1 error rate.

time-frequency pixels, FDR corrected). The largest magnitude of the effect in this direction occurred between 5 and $9 \mathrm{~Hz}$, with relatively greater oscillatory power during unsuccessful encoding between 500 and 1200 msec after stimulus presentation. By contrast, in the high gamma frequency range, there was a significant increase in oscillatory power during successful encoding - a positive subsequent memory effect $(t(61)>2.99)$. Gamma power during successful encoding (especially in the high gamma range, from 70 to $180 \mathrm{~Hz}$ ) reached a maximum at $1500 \mathrm{msec}$ after the onset of a study item (Figure 2). Time-frequency pixels that survived FDR correction (FDR of 0.05) are shown in Figure 2 . We next analyzed the subsequent memory effect from 73 hippocampal contacts inserted into the 14 partici- pants who also had posterior cingulate contacts. The positive effect in the gamma band (positive gamma SME) was robust in the hippocampus, with a persistent effect that survived FDR correction $(t(72)>3.14)$ across the first half of the time series (Figure 3). This finding is consistent with existing data (Lega et al., 2011). The subsequent memory effects within the posterior cingulate generally behaved similarly to those in the hippocampus: Thus, we observed a low-frequency power decrease and a high-frequency power increase accompanying successful item encoding. However, inspection of the time series data suggests that hippocampal gamma power remained elevated during the first 1000 msec after item presentation, whereas in the posterior cingulate, the gamma power maximum occurred 
later in the time series. We examined this observation statistically by calculating the difference in the mean $z$ value for the SME from the first half $(1-1000 \mathrm{msec})$ versus second half (1000-2000 msec) of the time series for PCC and hippocampal contacts and then comparing these two distributions. For the PCC, the early-late difference in SME $z$ value was -0.44 (late SME greater), whereas for the hippocampus, it was +0.77 ; these values differed significantly $(t(133)=3.11)$. This result confirms the impression from Figure 2 that the PCC gamma power SME occurs later in the time series than does the corresponding hippocampal effect.

We examined the power data in another way by constructing histograms of the total number of electrodes that exceeded the Type 1 error rate for the statistical test between recalled and nonrecalled items (5\%) at each time-frequency pixel and then tested this fraction of electrodes against the number expected by chance (binomial test). These data are shown alongside the statistical test comparing $z$ values for the analyses of differences in oscillatory power in Figures 2 and 3.

\section{Hippocampal-PCC Synchrony during Item Encoding}

We next examined encoding-related phase synchrony between the posterior cingulate and hippocampus. Across 14 participants with contacts in both locations, we identified 123 ipsilateral pairs between the two structures (including both anterior and posterior hippocampal contacts). Encoding-related modulation of phase synchrony presented a number of interesting parallels with the power analysis. Connectivity centered at $3 \mathrm{~Hz}$ was significantly greater during unsuccessful item encoding ( $p<.05$, FDR correction after $t$ test with shuffle procedure to test for differences between distributions of synchrony test statistics) for nearly six full cycles of the oscillation. Significantly greater synchrony for later nonrecalled study items was also present $1 \mathrm{sec}$ after item presentation centered at $9 \mathrm{~Hz}$. This implies that the oscillatory power differences we observed for this frequency are associated with desynchronization between the hippocampus and posterior cingulate (and potentially

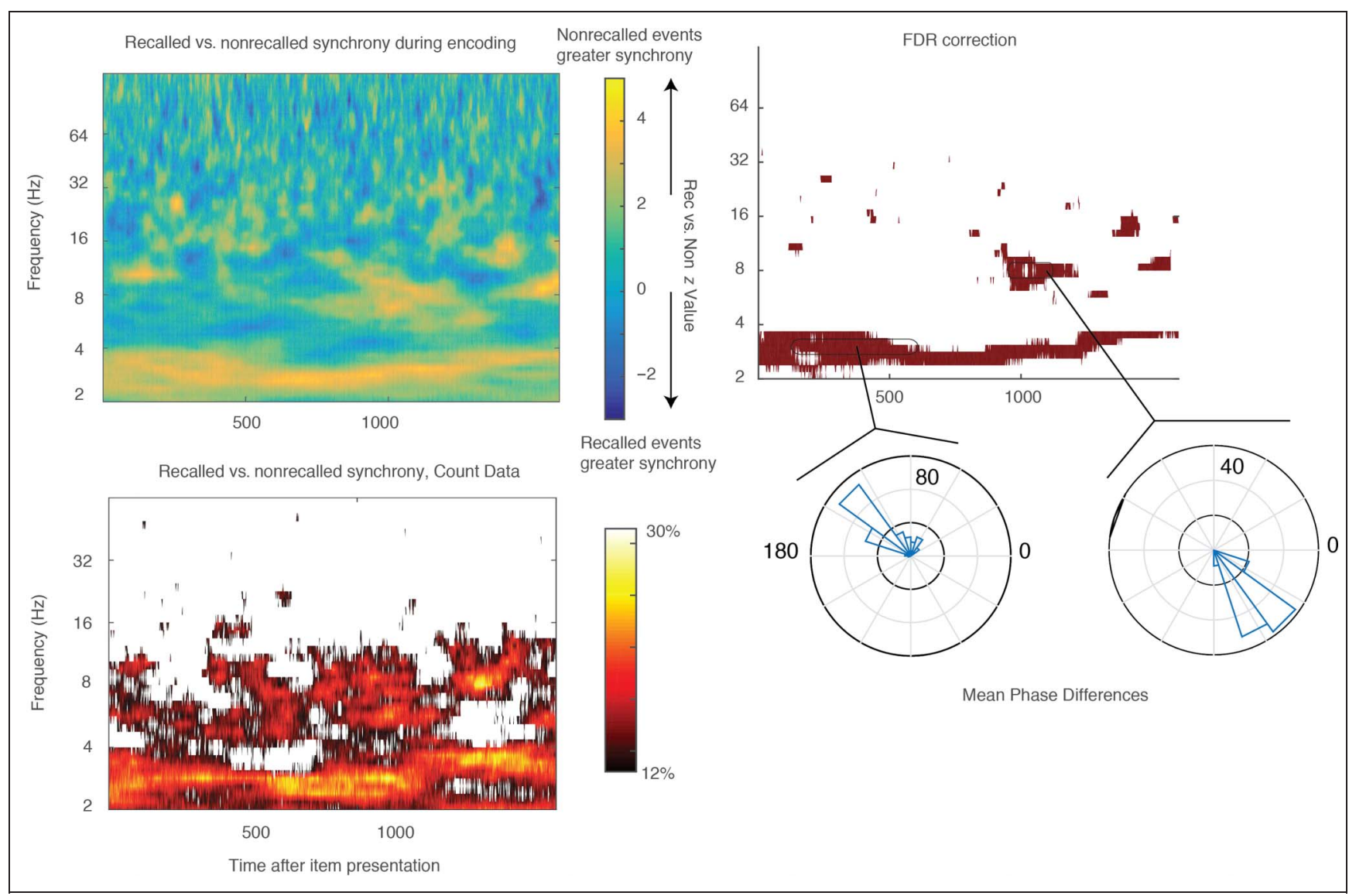

Figure 4. Hippocampal-posterior cingulate synchrony during item encoding. Top plot shows synchrony during item encoding across all 14 participants; there is a strong effect in the 2- to 4-Hz frequency range, with greater synchrony during unsuccessful item encoding, that persists across the time series. The difference in power observed at $1300 \mathrm{msec}$ (Figure 2) is associated with a drop in hippocampal-PCC synchrony at this frequency. Bottom left plot shows histogram of pixels at which the difference between recalled and nonrecalled events exceeds a difference in magnitude of the PLS of 3. Bottom right plots show a histogram compiling the circular mean phase difference between the hippocampus and PCC across events at each time point for two full cycles of the oscillation in the time window shown. Scales are different because the number of samples in two cycles is different for oscillations of different frequencies. 


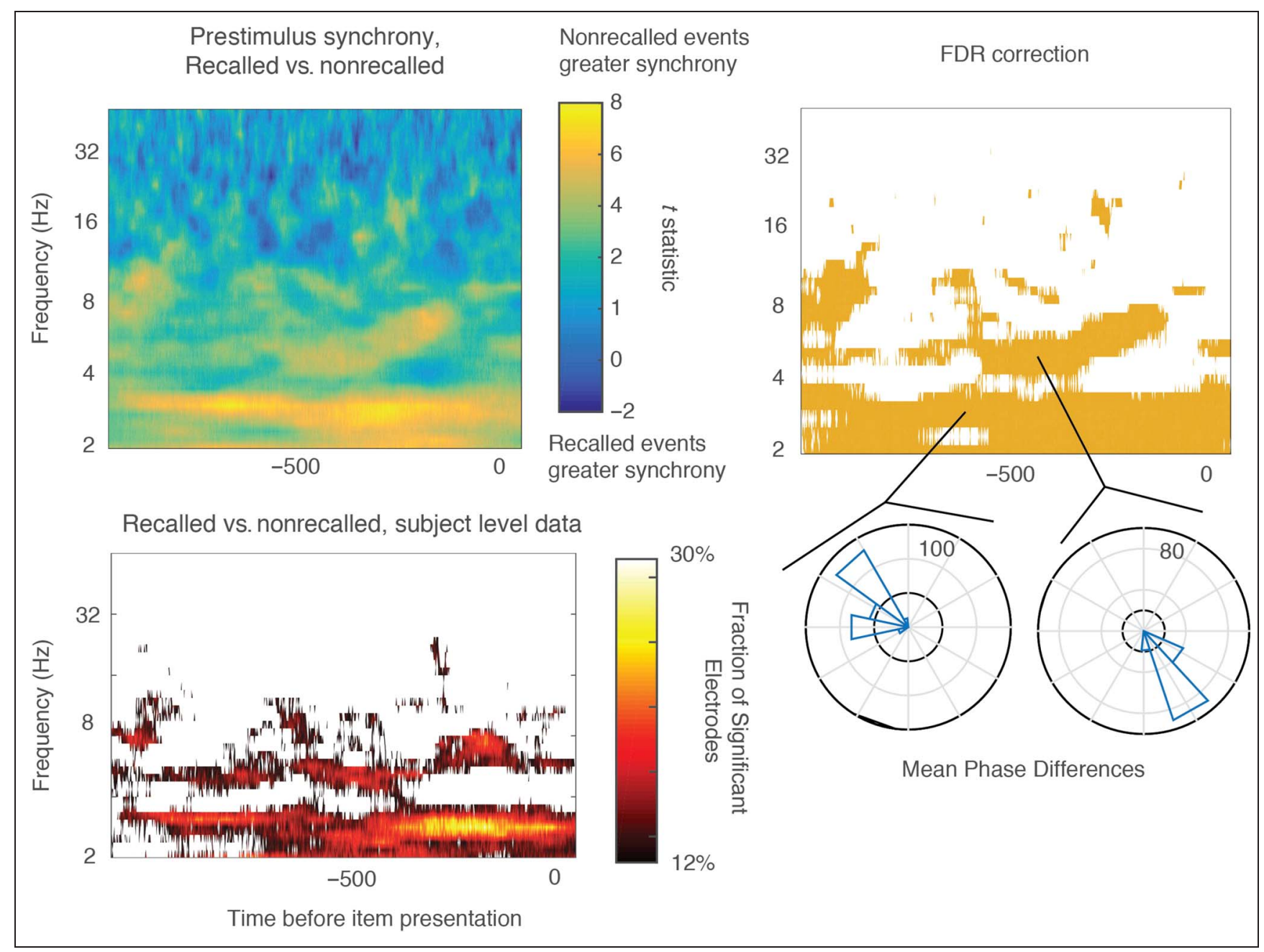

Figure 5. Hippocampal-posterior cingulate synchrony before presentation of memory items. Top left plot shows the time-frequency plot of synchrony difference between recalled and nonrecalled events relative to moment of item presentation; top right plot shows the FDR-corrected plot. Bottom left plot shows the histogram compiling the fraction of electrodes exhibiting a significant memory effect at each time-frequency pixel.

wider brain areas, although see Figure 6, as the power and synchrony effects may be driven by complementary populations of electrodes). A connectivity SME at this 9-Hz frequency lasted for approximately three cycles of the oscillation, with greater synchrony for nonrecalled study items (see FDR-corrected plot, Figure 4). For the histogram of time-frequency pixels comparing effects, it is not possible to conduct a statistical test within electrode pairs between recalled and nonrecalled events using the PLS method. We therefore counted the number of electrodes at each pixel that exhibited a difference of 3 for the PLS $z$ value from the shuffle procedure between recalled and nonrecalled events to produce across-participant data analogous to the histogram in the power analysis in Figure 2 (bottom right).

The modulation of hippocampal-cingulate synchrony centered at $3 \mathrm{~Hz}$ that exhibited an SME during item encoding began early in the time series and persisted thereafter, suggesting that it might reflect a prestimulus effect that extended into the post-item epoch. We compared oscillatory synchrony using the same method as described above and tested for a prestimulus subsequent memory effect, examining the $1000 \mathrm{msec}$ immediately before the appearance of a study item. As was the case for the poststimulus effect, a significant difference in synchrony persisted for a full cycle of the $3-\mathrm{Hz}$ oscillation, with greater synchrony preceding the onset of subsequently forgotten memory items (corrected $p<.05, t$ test with shuffle procedure comparing $z$ value distributions for 123 electrode pairs, recalled versus nonrecalled items; Figure 5).

\section{Correlation between Power and Connectivity Memory Effects}

We sought to examine our results for the interaction between oscillatory power and synchrony. The relationship between memory-related changes in power and in synchrony is complex; there is a "chicken and egg" problem in that synchronization can drive increases in power, but there is a theoretical concern that increases in power can 
artificially drive the measure of synchrony because of improved phase resolution for a given signal. We examined this issue by looking for a correlation (Spearman correlation) between the magnitude ( $z$ value) of the oscillatory power subsequent memory effect and the connectivity-related subsequent memory effect (difference in PLS $z$ value for each electrode). The results are shown in Figure 6, averaged over the 2.5- to 5- Hz slow theta frequency range and 5- to $10-\mathrm{Hz}$ range that also exhibits a negative SME in the PCC and hippocampal data. Overall, the magnitude of the correlation is not strong, with the largest $r$ value being .33 for the slow-theta oscillation in the PCC $(p=.09)$. However, there is a slight difference between this oscillation and the 5- to $10-\mathrm{Hz}$ oscillation, in that the correlation is positive in both hippocampus and PCC in the slow-theta range ( $r$ s $=.33$ and .26) and slightly negative in the $5-$ to $10-\mathrm{Hz}$ range ( $r \mathrm{~s}=-.17$ and -.02). This implies that electrodes exhibiting a slowtheta power decrease also exhibit relatively greater desynchronization during successful encoding at this range, but for the 5- to 10-Hz oscillation, the connectivity and power SME effects are driven by complementary populations of electrodes. Overall, the modest correlation and difference among frequency bands imply that power differences alone do not explain the connectivity memory effects, although power decreases may be partly driven by desynchronization between the hippocampus and PCC.

\section{PCC and Hippocampal Power during Item Retrieval}

We also compared oscillatory activity between the hippocampus and posterior cingulate during item retrieval. Results are shown in Figure 7 . We began by first analyzing power within each structure separately during the 1500-msec epoch preceding the vocalization of a retrieved memory item using established methods to avoid overlapping with activity elicited by temporally adjacent responses (Burke et al., 2014). We limited our analysis to correct responses (excluding extra list intrusions and PLIs). The retrieval phase in the free recall task was noisier than in the encoding phase, and our method for isolating vocalization events further reduced statistical power, but existing data positing a role for the cingulate during retrieval strongly motivated the analysis. We compared oscillatory power during retrieval with a "baseline" created by randomly selecting 1500-msec epochs of time from the retrieval phase and averaging power across the time series to create a dummy distribution of baseline power values to statistically compare with the true retrieval events. This method

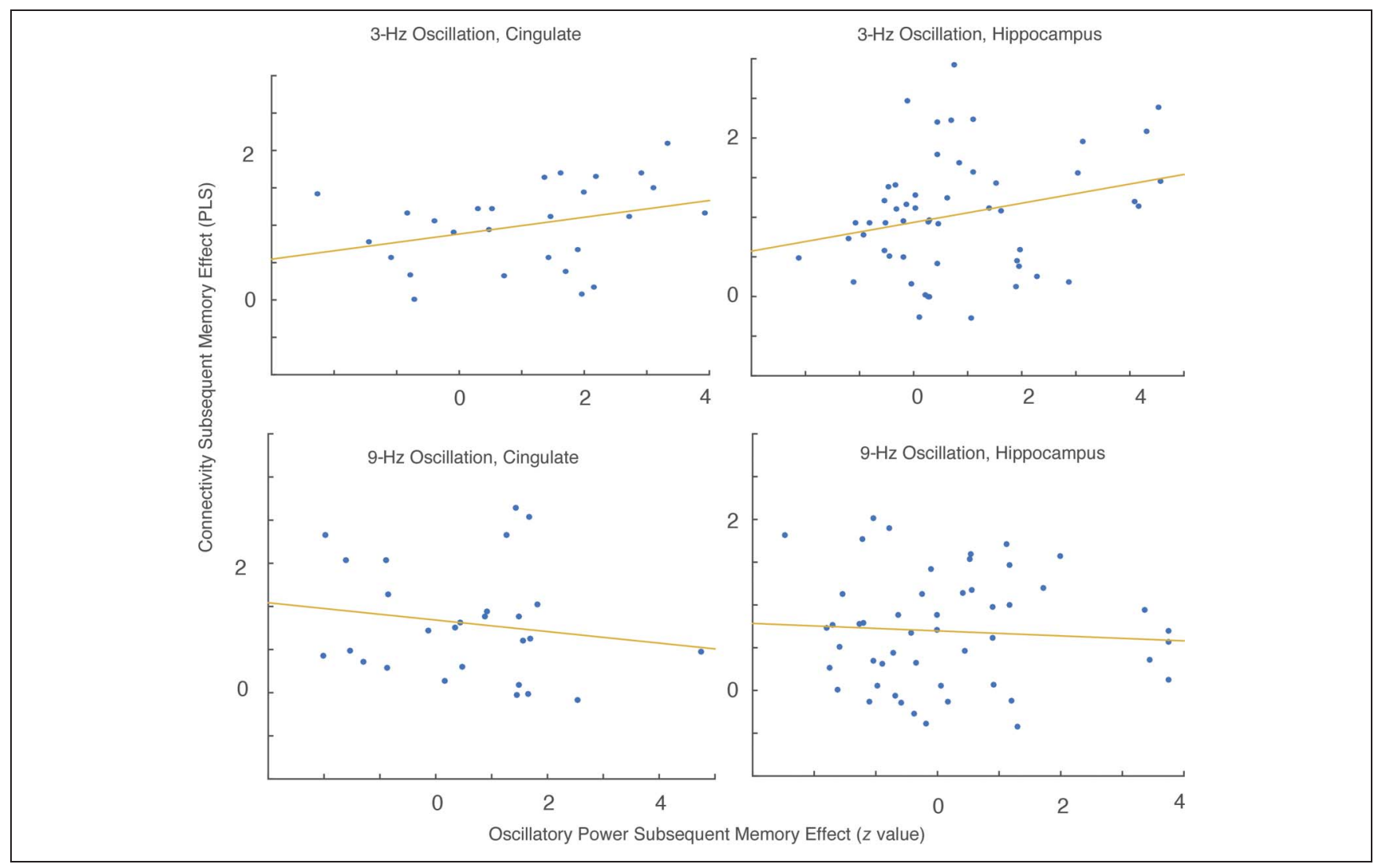

Figure 6. Correlation between oscillatory power and synchrony during encoding. Top row shows correlation between the synchrony and power subsequent memory effects for the posterior cingulate $(r=.33, p=.09)$ and hippocampus $(r=.26, p=.07)$ for the 3-Hz oscillation across the time series. Synchrony values for all electrode pairs were averaged for each cingulate or hippocampal electrode, respectively. Correlation values for the 5 - to $10-\mathrm{Hz}$ oscillation for the posterior cingulate $(r=-.17, p=.40)$ and hippocampus $(r=-.02, p=.90)$ are shown in the bottom row. 


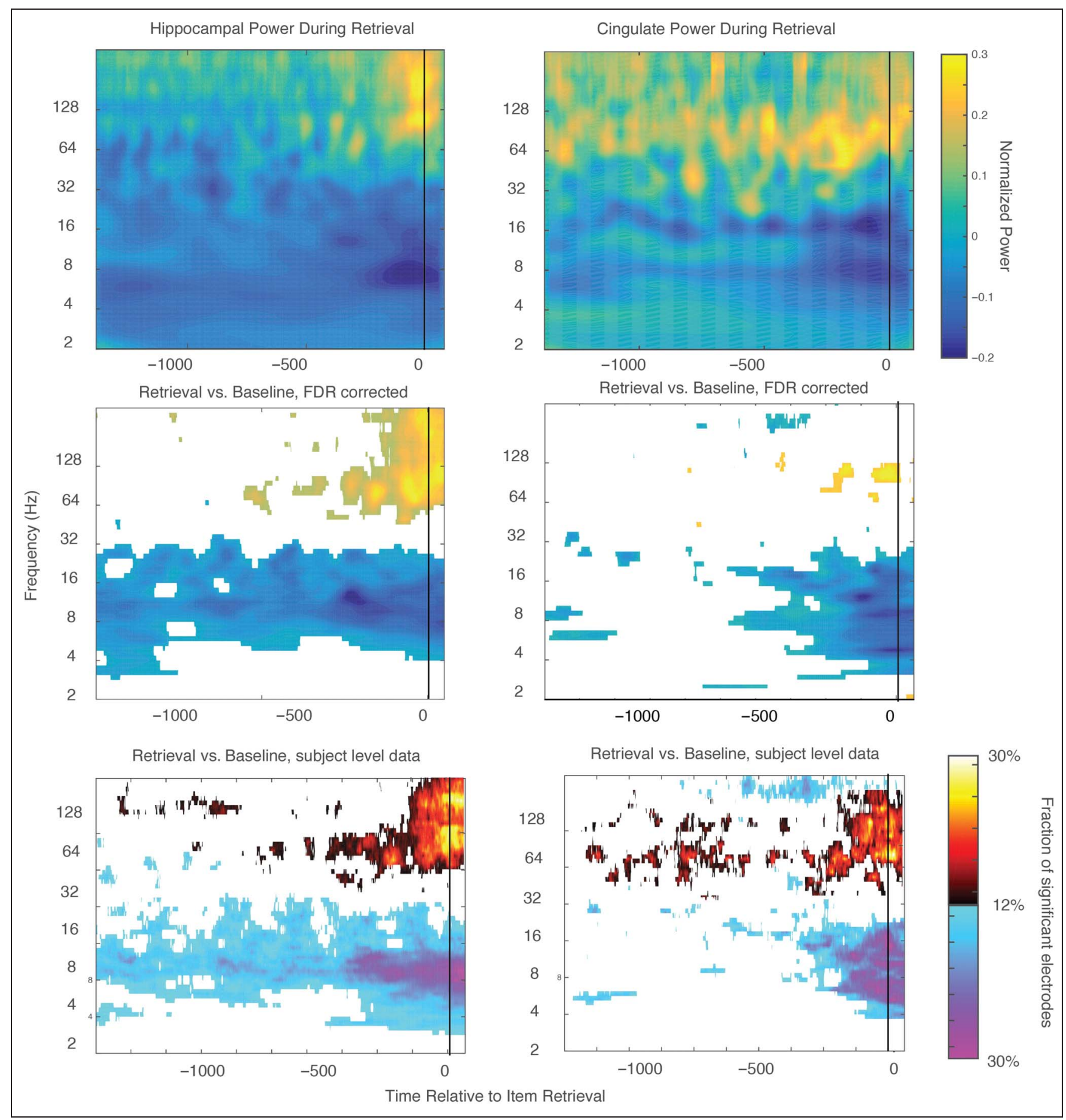

Figure 7. Oscillatory power differences in hippocampus and PCC during item retrieval. Top plots show average normalized power across all participants during 1500-msec window preceding vocalization of a retrieved memory item for the hippocampus (left) and posterior cingulate (right). Power increases in the hippocampus reach a rapid crescendo immediately before retrieval, whereas in the posterior cingulate, it occurs steadily throughout the time window. Middle plots demonstrate FDR-corrected time-frequency plots for statistical test between events preceding item retrieval and a distribution of time segments randomly drawn from the retrieval period. Bottom row shows histogram data.

allowed us to use a time window long enough to analyze oscillatory properties of the "slow-theta" oscillations (2.5$5 \mathrm{~Hz}$ ) previously observed in the hippocampus during retrieval. Overall, the pattern we observed in the hippocampus was consistent with previous reports (Lega et al., 2011), with a relatively narrow (in the frequency domain) decrease in power in the 5 - to $15-\mathrm{Hz}$ range (spanning theta and alpha bands) that reached a maximum immediately before the response vocalization (local minimum calculated across band power) but is robust across the time series. This power decrease occurred simultaneously with a broad gamma band power increase (from 40 to $200 \mathrm{~Hz}$ ) that also reached a peak before vocalization of the response but starts over $200 \mathrm{msec}$ earlier in the time series 
(maximum for average gamma power at $5 \mathrm{msec}$ before vocalization; Figure 7, top row). The power changes in the gamma range preceding vocalization in the hippocampus were quite robust, surviving FDR correction for the entire time series for the 5- to $15-\mathrm{Hz}$ oscillation and for $200 \mathrm{msec}$ for the gamma effect. The lower gamma effects (40-70 Hz) persist for longer, nearly $500 \mathrm{msec}$. These effects are also preserved in the histogram data (Figure 7, bottom row).

We performed the analogous analysis for the posterior cingulate electrodes. The power analysis revealed a persistent increase centered in the lower portion of the gamma band (below $100 \mathrm{~Hz}$ ) during item retrieval compared with baseline. The gamma band power increase in the posterior cingulate was significantly greater than the baseline retrieval epochs (randomly drawn 1500-msec segments from within the retrieval epoch, as above), surviving FDR correction for time-frequency points from $500 \mathrm{msec}$ before vocalization (corrected $p<.05 \mathrm{rank}$ sum test with shuffle procedure, FDR corrected, and inset plot in Figure 7). The low-gamma effect reached a maximum of $75 \mathrm{msec}$ before vocalization and survives FDR correction up to $200 \mathrm{msec}$, although it was present across the time series (see Figure 7, inset plots on the right). There was a concomitant decrease in low-frequency power between 5 and $15 \mathrm{~Hz}$ (Figure 7, top row, inset plots) that is strongest in the $500 \mathrm{msec}$ before item retrieval where it survives FDR correction. Histogram data were similar. These data all highlight a low-versus-high gamma distinction, with oscillations below $100 \mathrm{~Hz}$ exhibiting a persistent power increase relative to baseline, but above $130 \mathrm{~Hz}$, there is a relative power decrease between 400 and $500 \mathrm{msec}$ before item vocalization. This is in contrast to the encoding data, during which the gamma effect is strongest above $100 \mathrm{~Hz}$.

\section{Hippocampal-PCC Synchrony during Item Retrieval}

We also examined cingulate-hippocampal synchrony preceding item recall. As above in the power analyses, for statistical comparison, we randomly selected 1500-msec epochs from the retrieval period, calculated synchrony at each time point, and averaged the resultant $z$ values across the time series to provide a distribution for comparison in time-frequency space. This distribution of randomly drawn values was compared with the distribution of synchrony $z$ values for all electrode pairs (Figure 8). Synchrony at $2-3 \mathrm{~Hz}$ was significantly greater than the randomly selected synchrony values during the $500 \mathrm{msec}$ immediately preceding item retrieval, consistent with the timing of the strongest changes in oscillatory power. At $9 \mathrm{~Hz}$, there is an increase in synchrony earlier in the time series (before $500 \mathrm{msec}$ ) that survives FDR correction as well; there appears to be a subtle shift in the oscillatory pattern before and after $500 \mathrm{msec}$, by which 9-Hz synchrony switches to a stronger lower-frequency effect im- mediately before vocalization. There is not a significant power difference at this frequency at this point in the time series. Synchrony at this frequency reached a peak for around one cycle before vocalization of a retrieved item, where it survived FDR correction (corrected $p<$ $.05, t$ test with shuffle procedure comparing retrieval synchrony with baseline average synchrony). Although both the posterior cingulate and hippocampus exhibited gamma band power increases before item vocalization, there was no significant synchrony in this frequency range. Overall, the decrease in synchrony observed during successful encoding (at both 9 and $3 \mathrm{~Hz}$ ) is reversed during item retrieval, in which increases in synchrony appear to occur at these frequencies (although at different points in time) before a retrieved memory item is vocalized. There is evidence for similar connectivity changes for other parietal cortical locations in other memory tasks, which we address below.

\section{PCC Phase-Amplitude Coupling during Item Encoding}

We examined our encoding data for evidence of phaseamplitude coupling in the posterior cingulate. We quantified the PAC magnitude for three phase-providing bands (2.5-5, 5-10, and 11-14 Hz) and two power-providing bands (35-70 and 70-150 Hz) at each electrode separately during successful and unsuccessful encoding events and then compared the recalled and nonrecalled distributions of resulting test statistics across electrodes. Figure 9 shows the results. For none of the frequency bands was the phase-amplitude coupling (PAC) subsequent memory effect significant, although there is an interesting reversal in the direction of PAC for low versus high gamma. The phase preference for PAC did not strongly differ with encoding success (inset rose plots in Figure 9).

\section{DISCUSSION}

\section{PCC during Item Encoding}

We observed a significant low-frequency decrease and gamma band increase in power in the PCC during successful versus unsuccessful item encoding. This pattern matched that of the hippocampus in the frequency domain (lowfrequency power decrease, gamma band power increase during successful encoding), but it occurred later in the time domain (see Figures 2 and 3). The electrodes in our study were implanted directly within the posterior cingulate (and hippocampus; see localization in Figure 1) rather than placed on the brain surface in the interhemispheric fissure, making it unlikely that the signal we report was significantly contaminated by input from the visual cortex, precuneus, or other adjacent gray matter in this area. A novel aspect of our data is the simultaneous recording of PCC and hippocampal activity, which allowed us to identify a subsequent memory effect in the synchrony 


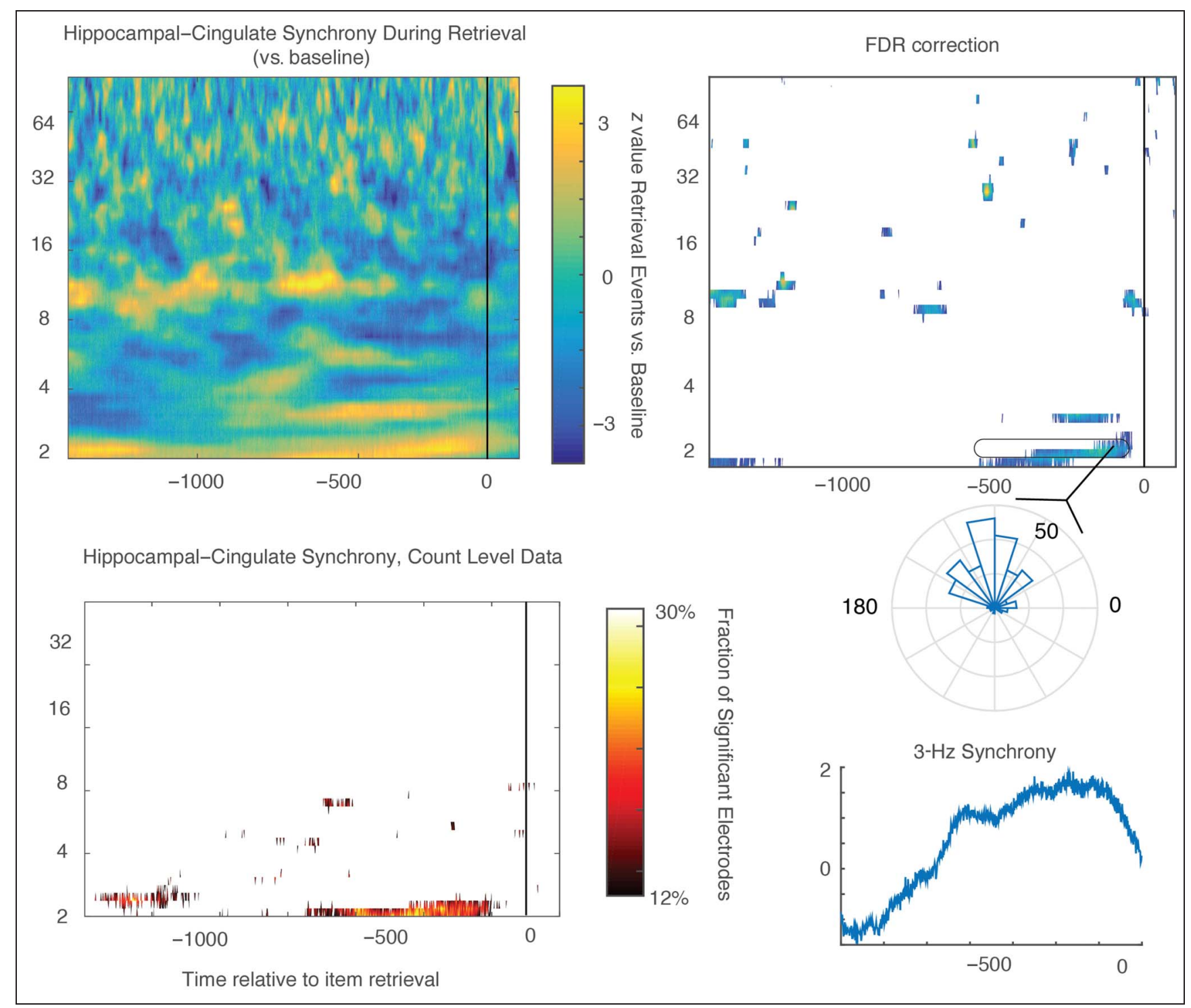

Figure 8. Hippocampal-posterior cingulate synchrony during item retrieval. Top plot shows pattern of connectivity differences during retrieval versus baseline distribution; right side shows FDR-corrected plot. Bottom left time-frequency plot shows the histogram data with time-frequency pixels for count of electrodes exhibiting a difference of 3 for the magnitude of PLS values drawn from recalled and nonrecalled events. Top inset plot is a histogram showing the mean phase difference across electrode pairs between hippocampus and PCC for the 3-Hz oscillation for two full oscillatory cycles during the period of significant synchrony as reflected in the time-frequency plot. Bottom inset plots show the time course of 3-Hz synchrony for retrieval events between the structures.

domain (along with oscillatory power changes) between these structures. The synchrony behavior was different for the sub-4-Hz oscillations and those above $4 \mathrm{~Hz}$; the latter exhibit desynchronization mainly in the second half of the time series, whereas the $3-\mathrm{Hz}$ oscillation was desynchronized during successful encoding throughout the time series (Figure 4). A 3-Hz desynchronization pattern was also present as a prestimulus effect (Figure 8), with decreased hippocampal-PCC synchrony immediately before the presentation of later remembered study items. Oscillatory power (along with connectivity) in the 2 - to $4-\mathrm{Hz}$ frequency range exhibited a negative SME (power decrease during successful encoding) in the PCC during the second half of the time series. These findings join the results of other investigations of human episodic and spatial memory that identify correlations between oscillatory changes in this frequency range and successful memory encoding (Watrous, Lee, et al., 2013; Lega et al., 2011; Watrous et al., 2011). Theta activity has been observed in the rodent cingulate cortex, which can be synchronous with hippocampal theta or independently generated (Talk, Kang, \& Gabriel, 2004; Leung \& Borst, 1987). The identification of a power and connectivity SME centered at $3 \mathrm{~Hz}$ in the PCC may lend support to the idea that human $3-\mathrm{Hz}$ oscillations exhibit properties akin to rodent theta activity that is critical for memory processes (Jacobs, 2014); the existence of multiple $3-\mathrm{Hz}$ generators including in the PCC (as suggested in animal studies) may be consistent with different 
properties that have been observed for oscillations at this frequency. We did not however observe a power increase at this frequency in the PCC, as has been observed for a subset of hippocampal electrodes in previous studies (Lega et al., 2011; Watrous et al., 2011). A direct comparison of hippocampal connectivity properties with mnemonically relevant locations such as the rhinal cortex and lateral temporal region would help elucidate how this oscillation acts to integrate feature and context information for episodic memory. The broader sub-15-Hz power decrease and associated desynchronization include a strong component in the 5- to 9-Hz frequency range at the upper edge of the traditional 4- to $8-\mathrm{Hz}$ theta band. Analysis of hippocampal power suggested that this oscillation and the $3-\mathrm{Hz}$ oscillation have different properties (Lega et al., 2011); however, we observed that an oscillation centered at $9 \mathrm{~Hz}$ exhibits a (weaker) increase in synchrony during retrieval along with the 3-Hz oscillation. This observation may support the idea of complementary theta oscillations centered at different frequencies with some analogy in the rodent literature (Montoya \& Sainsbury, 1985).

The oscillatory pattern in the PCC during encoding (low-frequency power decrease, with gamma band power increase, similar to the hippocampus) is indicative of active information processing (Fries, 2009), suggesting that the fMRI BOLD reduction typically observed for successful encoding in several brain structures (including the PCC) during memory tasks may in some cases be indicative of cognitive engagement (Bird, Keidel, Ing, Horner, \& Burgess, 2015; Ekstrom, Suthana, Millett, Fried, \& Bookheimer, 2009); however, the BOLD-electrophysiology relationship often differs region by region and in the frequency of maximum correlation between modalities (Ekstrom, 2010). The present encoding pattern is consistent with some

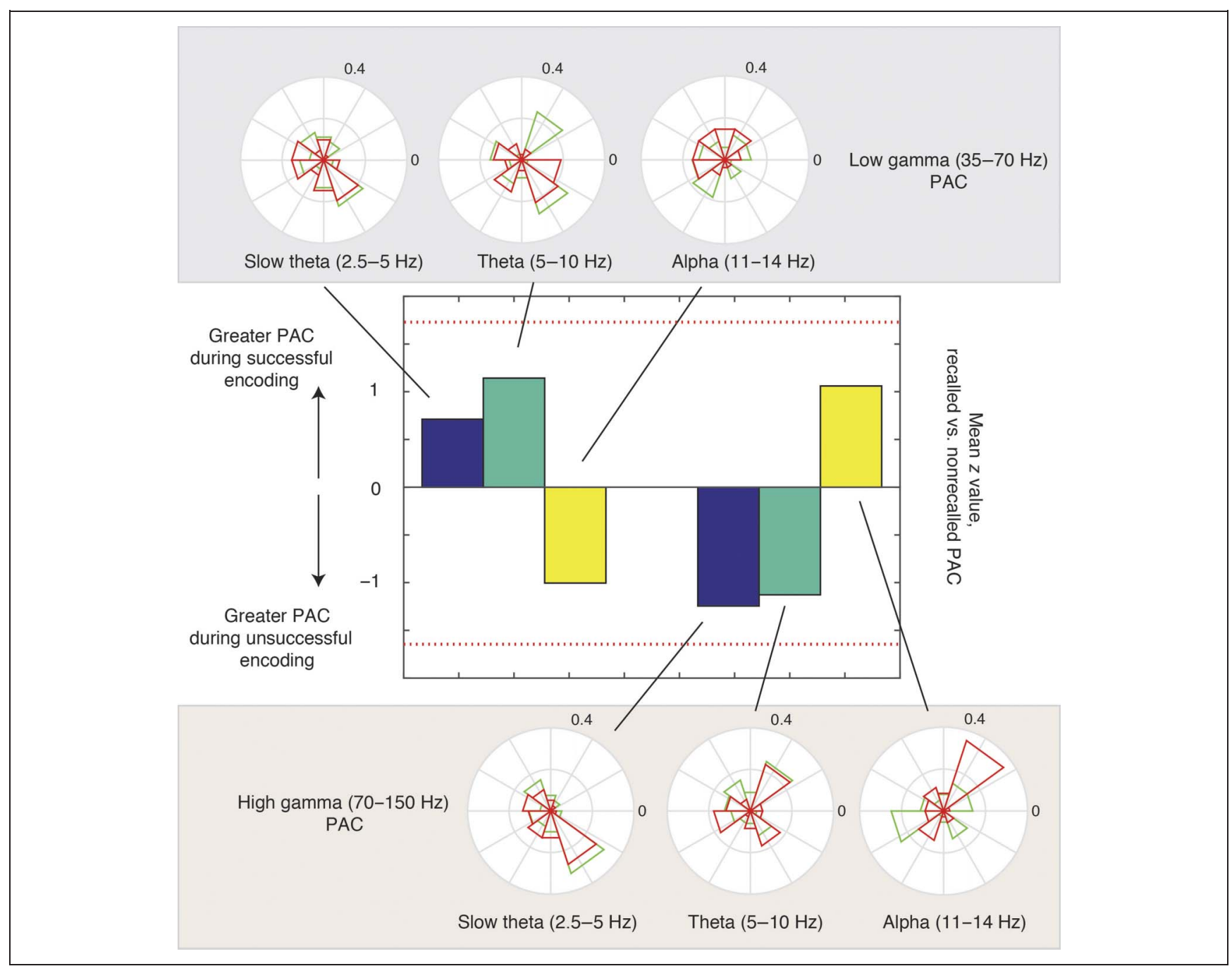

Figure 9. Phase-amplitude coupling in the posterior cingulate. Bar plot shows the magnitude of the subsequent memory effect ( $t$ statistic) comparing PAC for recalled and nonrecalled events, organized by phase-providing frequency band (each bar: slow theta, theta, alpha) and powerproviding frequency band (low gamma in the left group, high gamma in the right group). The red line indicates the $p=.05$ threshold (across electrodes) for a PAC-related subsequent memory effect. Surrounding rose plots show the distribution of the preferred phase for coupling at each electrode compiled into histograms, with red color indicating nonrecalled events and green indicating recalled events. Scale for rose plots is the fraction of total electrodes. 
aspects of the "information via desynchronization hypothesis," the proposal that power decreases across several frequency ranges may be associated with the encoding of different features of an event (Hanslmayr \& Staudigl, 2014; Hanslmayr, Staudigl, \& Fellner, 2012). As initially articulated, the hypothesis sought to account for the observation of widespread cortical 9- to $20-\mathrm{Hz}$ range power decreases during episodic memory encoding, with "desynchronization" referring to local cortical desynchronization manifest as a power decrease. Our data further suggest that 4- to 9-Hz power changes in the hippocampus and PCC may be driven by widespread desynchronization at these frequencies during memory encoding. This same frequency band has been reported to exhibit modulation of connectivity between the hippocampus and temporal cortex during item encoding (Lega et al., 2011), and power decreases in this range have been associated with fMRI BOLD decreases (Hanslmayr et al., 2012).

\section{PCC during Item Retrieval}

Our behavioral paradigm cannot disambiguate neural activity related to memory retrieval from that related to speech preparation, but existing anatomical and functional data for the role of the PCC do not suggest that speech planning would contribute strongly to observed oscillatory activity in the PCC (Leech \& Sharp, 2014). Given a larger sample size, the number of incorrect retrieval events would perhaps allow a comparison of oscillatory activity associated with successful versus unsuccessful recall attempts. This caveat notwithstanding, the retrievalrelated oscillatory patterns reported here may have interesting implications for proposals about the role of the PCC in episodic memory. Notably, we observed a persistent gamma band power increase and a 4- to 9-Hz power decrease during item retrieval (Figure 7), which may be an electrophysiological correlate of the retrieval-related BOLD signal increase seen during this period in existing fMRI investigations (for reviews, see Rugg \& Vilberg, 2013; Kim, 2010; and Spaniol et al., 2009). High gamma changes in mesial parietal regions during item retrieval have been observed for autobiographical memory items (Foster et al., 2012). The correlation between BOLD signal changes and iEEG patterns likely depends on the specific brain region in question and, most likely, the nature of the cognitive task, but it is interesting that our observations of gamma activity during retrieval (with a predominant effect in lower gamma ranges) match the existing BOLD data (decrease for encoding, increase for retrieval; see Introduction) better than during encoding when the gamma effect occurs in a slightly higher frequency range. The generalizability of this effect for adjacent brain areas such as the precuneus is an interesting question for future research. Analysis of the contribution of the precuneus to a wider memory retrieval network has been attempted for intracranial data (Watrous, Lee, et al., 2013); Watrous et al. discovered that connectivity for the precuneus (and the lateral parietal cortex) with the parahippocampus increases during successful versus unsuccessful item retrieval. This was stronger for spatial than temporal item context in their paradigm. These results were strongest at $8 \mathrm{~Hz}$ for the precuneus, although effects were present more diffusely between 1 and $10 \mathrm{~Hz}$. Our data are generally consistent with these findings in that we observed increased connectivity during item retrieval centered at $3 \mathrm{~Hz}$, although the previous work does not include the PCC directly. The results examined by Watrous et al. suggest that similar patterns of increased temporal connectivity are observed for the PCC as well as extra-PCC parietal lobe regions; analyses examining PCC-hippocampal along with PCC-parahippocampal gyrus connectivity during both encoding and retrieval will permit a more direct comparison with the spatial memory paradigm employed in their paper. This comparison of temporal connectivity across different memory paradigms is especially interesting in light of the strong findings identified in a recognition memory paradigm for the contributions of high gamma activity in the lateral parietal cortex (Gonzalez et al., 2015). Differences in the frequency preference for gamma range activity in encoding versus retrieval for episodic memories (to compare with these interesting recognition data) along the intraparietal sulcus may shed light on our findings with different effects in the PCC above and below $100 \mathrm{~Hz}$ during retrieval.

The timing of the hippocampal retrieval-related gamma power change, with a narrow increase in broadband gamma power $250 \mathrm{msec}$ before item retrieval, raises the possibility that the gamma power changes reflect ripple activity (150- to 300-Hz gamma activity of extremely short duration observed in the hippocampal local field potential in rodent data). Rodent hippocampal studies suggest that item retrieval in maze navigation (place cell recapitulation) is accompanied by $150-$ to $300-\mathrm{Hz}$ ripple activity (Carr, Jadhav, \& Frank, 2011). The duration of hippocampal ripples is around $150 \mathrm{msec}$. Hippocampal retrieval-related gamma activity across longer time scales in the high gamma range would be less consistent with a possible contribution of ripples to gamma power changes. The timing of PCC retrieval effects occur over a longer segment of the time series than for the hippocampus. Identifying ripples specifically using high impedance recording electrodes would more definitively characterize whether the gamma changes we observe are attributable to ripple activity, but our observed pattern is perhaps more consistent with a hypothesized role for ripples in the human hippocampus than if we had observed a power change spread over $1000 \mathrm{msec}$, for example.

\section{Mnemonic Role of the PCC}

The similarity in activity between encoding and retrieval that we observed is most consistent with prior reports that brain oscillatory patterns that occur during encoding are recapitulated during retrieval (Manning, Polyn, Baltuch, 
Litt, \& Kahana, 2011), although these previous studies did not include the posterior cingulate or adjacent mesial parietal regions in the analysis. The recapitulation of encoding-related neural activity during retrieval has been hypothesized to reflect "mental time travel" that is a hallmark of episodic memory that includes retrieval of perceptual, conceptual, and contextual features of a study event (retrieved context theory; Polyn \& Sederberg, 2014). Within this wider theory, there is evidence that the contribution of the PCC to episodic memory is in support of the representation of contextual information. Existing data link PCC activity to the encoding of emotional context, and data demonstrating specific PCC activation during the retrieval of autobiographical memories of familiar places (vs. objects) may argue in favor of PCC contribution to representing specific features of context (Sugiura, Shah, Zilles, \& Fink, 2005; Smith, Henson, Dolan, \& Rugg, 2004; Maddock, Garrett, \& Buonocore, 2003). Alternatively, data suggesting that the PCC is activated during both the retrieval of autobiographical memories and imagining future situations (Leech \& Sharp, 2014) and data suggesting that the magnitude of PCC activation is correlated with the amount of contextual information (rather than the content) that is retrieved (Thakral, Wang, \& Rugg, 2015; Leiker \& Johnson, 2014) imply that, along with other members of the "core recollection network," the PCC serves a more general role than the representationspecific contextual information (Rugg \& Vilberg, 2013). The PCC may instead support the integration of contextual information necessary to place item and context details into a common episodic representation. Such a theory may account for active PCC information processing during both encoding and retrieval, with matching gamma band power increase and low-frequency power decrease, as we observed. Further testing of this view could include different behavioral paradigms that make different contextual demands for encoding and retrieval. Within the free recall paradigm, an examination of PLIs (erroneous contextual information) versus correct retrieval may also provide insight, along with a comparison of PCC activity (within participants) for retrieved items that exhibit temporal versus semantic clustering patterns (Manning, Sperling, Sharan, Rosenberg, \& Kahana, 2012).

Our data are, however, also consistent with other views of PCC function during item retrieval. The "attention to internal representations" hypothesis proposes that the PCC is involved in shifting internal attentional resources to representations from external stimulus events to retrieved memory representations (Cabeza et al., 2008). The synchronous 3-Hz oscillation in the PCC and hippocampus has properties that fit this model: Hippocampal synchrony decreases during successful encoding (Figure 4), when attentional resources are presumably directed toward external stimuli, but it increases only before vocalization of a retrieved memory item (Figure 7). Synchrony of $3 \mathrm{~Hz}$ activity between the hippocampus and lateral parietal cortex has been observed to increase as a prestimulus effect, and the spatial distribution and timing of this effect overlapped with areas thought to participate in attention networks, leading the authors to hypothesize that 3-Hz synchrony is a marker of a global attention signal affecting memory performance (Haque, Wittig, Damera, Inati, \& Zaghloul, 2015). Hippocampal-PCC 3-Hz synchrony may underlie a functionally different attentional signal. The prestimulus decrease in synchrony we observed for successful encoding also fits this hypothesis, as attention is directed toward a novel memory item. The gamma band effect evident during encoding does not fit this hypothesis as neatly, however. Activity at different frequency ranges may support different but complementary functional roles for the PCC for memory.

Our results may be consistent with existing literature identifying a difference in midline parietal alpha oscillatory power $(8-12 \mathrm{~Hz})$ during internally versus externally directed attentional tasks (Aftanas \& Golocheikine, 2001). During encoding, we observed strong desynchronization for oscillations at or adjacent to this frequency range (5$10 \mathrm{~Hz}$ ) and then observed increased hippocampal-PCC synchrony centered at $9 \mathrm{~Hz}$ preceding item retrieval (Figure 5). However, the stronger effect in this regard is at $3 \mathrm{~Hz}$; in the period immediately preceding item retrieval, the 9-Hz effect is absent, and during this phase, there is a strong power decrease in both the PCC and hippocampus (Figure 7). The differential properties of slow-theta $(3 \mathrm{~Hz})$ and theta-alpha (5-14 Hz) oscillations during different attentional tasks may clarify how strongly the PCC is contributing to the previously observed alpha desynchronization for external attention. The differential properties of connectivity in the frequency and time domains we observed for 3- versus 9-Hz oscillations may reflect different spectral properties within similar anatomical networks for different features of memory (Ekstrom \& Watrous, 2014). Examining direct PCC recordings during spatial navigation, or comparing temporal versus semantic clustering features as described above, may elicit further evidence of "spectral fingerprinting" as described by Ekstrom and Watrous (2014) for spatial versus episodic memory. As they discuss, identification of connectivity between the hippocampus and parietal lobe structures may provide further evidence for the importance of phase coding to memory encoding and retrieval, although it is not clear how an oscillation with differential properties during these two phases of memory (as we observed for the 3-Hz oscillation) could contribute to phase coding (Watrous, Tandon, Conner, Pieters, \& Ekstrom, 2013). The prestimulus effects we observed in the beta frequency band (which were absent during encoding after item presentation and during retrieval) have been proposed as a part of top-down attentional control mechanisms in visual processing (Donner \& Siegel, 2011), especially as compared with lower-frequency desynchronization (reflecting distant connectivity) and highfrequency activity thought to be more indicative of the local encoding of information. Such a model may account for several features of parietal activity in episodic memory, 
although it was described more specifically for visual processing.

\section{Conclusion}

Our study utilized sEEG to obtain direct, simultaneous recordings from the hippocampus and PCC as patients performed an episodic memory task. We identify for the first time a subsequent memory effect in the PCC during encoding for both oscillatory power changes (including a gamma band power increase) and for connectivity with the hippocampus centered at $3 \mathrm{~Hz}$. We also identified increased synchrony at this frequency between the hippocampus and PCC immediately before vocalization of retrieved memory items, along with a gamma band effect that parallels what we observed during the encoding phase. The SME we observe during encoding is consistent with the information via desynchronization hypothesis for item representation, and the recapitulation of this pattern during item retrieval is consistent with current models positing context reinstatement in free recall. The findings suggest that the PCC plays an active role in both encoding and retrieval, possibly supporting the integration of contextual and item-specific representations into coherent episodes. Further investigations, incorporating the study of recall errors and disruptive stimulation, will test these hypotheses directly.

\section{Acknowledgments}

Funding was provided in part via a University of Texas Brain Seed grant no. 366582 .

Reprint requests should be sent to Bradley Lega, UT-Southwestern Medical Center, Neurological Surgery MS 8855, 5323 Harry Hines Blvd, Dallas, TX 75390, or via e-mail: bradlega@ gmail.com.

\section{REFERENCES}

Aftanas, L., \& Golocheikine, S. (2001). Human anterior and frontal midline theta and lower alpha reflect emotionally positive state and internalized attention: High-resolution EEG investigation of meditation. Neuroscience Letters, 310, 57-60.

Bird, C. M., Keidel, J. L., Ing, L. P., Horner, A. J., \& Burgess, N. (2015). Consolidation of complex events via reinstatement in posterior cingulate cortex. Journal of Neuroscience, 35, 14426-14434.

Burke, J. F., Sharan, A. D., Sperling, M. R., Ramayya, A. G., Evans, J. J., Healey, M. K., et al. (2014). Theta and high-frequency activity mark spontaneous recall of episodic memories.

Journal of Neuroscience, 34, 11355-11365.

Cabeza, R., Ciaramelli, E., Olson, I. R., \& Moscovitch, M. (2008). The parietal cortex and episodic memory: An attentional account. Nature Reviews Neuroscience, 9, 613-625.

Carr, M. F., Jadhav, S. P., \& Frank, L. M. (2011). Hippocampal replay in the awake state: A potential substrate for memory consolidation and retrieval. Nature Neuroscience, 14, 147-153.

Choo, I. H., Lee, D. Y., Oh, J. S., Lee, J. S., Lee, D. S., Song, I. C., et al. (2010). Posterior cingulate cortex atrophy and regional cingulum disruption in mild cognitive impairment and Alzheimer's disease. Neurobiology of Aging, 31, 772-779.

De Schotten, M. T., Dell'Acqua, F., Valabregue, R., \& Catani, M. (2012). Monkey to human comparative anatomy of the frontal lobe association tracts. Cortex, 48, 82-96.

Devinsky, O., \& Luciano, D. (1993). The contributions of cingulate cortex to human behavior. In B. A. Vogt \& M. Gabriel (Eds.), Neurobiology of cingulate cortex and limbic thalamus (pp. 527-556). Boston: Birkhäuser.

Donner, T. H., \& Siegel, M. (2011). A framework for local cortical oscillation patterns. Trends in Cognitive Sciences, 15, 191-199.

Ekstrom, A. (2010). How and when the fMRI bold signal relates to underlying neural activity: The danger in dissociation. Brain Research Reviews, 62, 233-244.

Ekstrom, A., Suthana, N., Millett, D., Fried, I., \& Bookheimer, S. (2009). Correlation between bold fMRI and theta-band local field potentials in the human hippocampal area. Journal of Neurophysiology, 101, 2668-2678.

Ekstrom, A. D., \& Watrous, A. J. (2014). Multifaceted roles for low-frequency oscillations in bottom-up and top-down processing during navigation and memory. Neuroimage, 85, 667-677.

Elward, R. L., \& Rugg, M. D. (2015). Retrieval goal modulates memory for context. Journal of Cognitive Neuroscience, 27, 2529-2540.

Fellgiebel, A., Müller, M. J., Wille, P., Dellani, P. R., Scheurich, A., Schmidt, L. G., et al. (2005). Color-coded diffusion-tensorimaging of posterior cingulate fiber tracts in mild cognitive impairment. Neurobiology of Aging, 26, 1193-1198.

Foster, B. L., Dastjerdi, M., \& Parvizi, J. (2012). Neural populations in human posteromedial cortex display opposing responses during memory and numerical processing. Proceedings of the National Academy of Sciences, U.S.A., 109, 15514-15519.

Foster, B. L., Kaveh, A., Dastjerdi, M., Miller, K. J., \& Parvizi, J. (2013). Human retrosplenial cortex displays transient theta phase locking with medial temporal cortex prior to activation during autobiographical memory retrieval. Journal of Neuroscience, 33, 10439-10446.

Foster, B. L., Rangarajan, V., Shirer, W. R., \& Parvizi, J. (2015). Intrinsic and task-dependent coupling of neuronal population activity in human parietal cortex. Neuron, 86, 578-590.

Fries, P. (2009). Neuronal gamma-band synchronization as a fundamental process in cortical computation. Annual Review of Neuroscience, 32, 209-224.

Gonzalez, A., Hutchinson, J. B., Uncapher, M. R., Chen, J., LaRocque, K. F., Foster, B. L., et al. (2015).

Electrocorticography reveals the temporal dynamics of posterior parietal cortical activity during recognition memory decisions. Proceedings of the National Academy of Sciences, U.S.A., 112, 11066-11071.

Greicius, M. D., Krasnow, B., Reiss, A. L., \& Menon, V. (2003). Functional connectivity in the resting brain: A network analysis of the default mode hypothesis. Proceedings of the National Academy of Sciences, U.S.A., 100, 253-258.

Hanslmayr, S., \& Staudigl, T. (2014). How brain oscillations form memories-A processing based perspective on oscillatory subsequent memory effects. Neuroimage, 85 , 648-655.

Hanslmayr, S., Staudigl, T., \& Fellner, M.-C. (2012). Oscillatory power decreases and long-term memory: The information via desynchronization hypothesis. Frontiers in Human Neuroscience, 6, 74.

Haque, R. U., Wittig, J. H., Damera, S. R., Inati, S. K., \& Zaghloul, K. A. (2015). Cortical low-frequency power and progressive phase synchrony precede successful memory encoding. Journal of Neuroscience, 35, 13577-13586. 
Jacobs, J. (2014). Hippocampal theta oscillations are slower in humans than in rodents: Implications for models of spatial navigation and memory. Philosophical Transactions of the Royal Society of London, Series B: Biological Sciences, 369, 20130304.

Kim, H. (2010). Dissociating the roles of the default-mode, dorsal, and ventral networks in episodic memory retrieval. Neuroimage, 50, 1648-1657.

Kubota, Y., Enatsu, R., Gonzalez-Martinez, J., Bulacio, J., Mosher, J., Burgess, R. C., et al. (2013). In vivo human hippocampal cingulate connectivity: A corticocortical evoked potentials (CCEPs) study. Clinical Neurophysiology, 124, 1547-1556.

Lachaux, J. P., Rodriguez, E., Martinerie, J., \& Varela, F. J. (1999). Measuring phase synchrony in brain signals. Human Brain Mapping, 8, 194-208.

Leech, R., \& Sharp, D. J. (2014). The role of the posterior cingulate cortex in cognition and disease. Brain, 137, 12-32.

Lega, B., Dionisio, S., Bingaman, W., Najm, I., \& GonzalezMartinez, J. (2015). The gamma band effect for episodic memory encoding is absent in epileptogenic hippocampi. Clinical Neurophysiology, 126, 866-872.

Lega, B., Jacobs, J., \& Kahana, M. (2011). Human hippocampal theta oscillations and the formation of episodic memories. Hippocampus, 22, 748-761.

Leiker, E. K., \& Johnson, J. D. (2014). Neural reinstatement and the amount of information recollected. Brain Research, 1582, 125-138.

Leung, L.-W., \& Borst, J. (1987). Electrical activity of the cingulate cortex: I. Generating mechanisms and relations to behavior. Brain Research, 407, 68-80.

Maddock, R., Garrett, A., \& Buonocore, M. (2001). Remembering familiar people: The posterior cingulate cortex and autobiographical memory retrieval. Neuroscience, 104, 667-676

Maddock, R. J., Garrett, A. S., \& Buonocore, M. H. (2003). Posterior cingulate cortex activation by emotional words: fMRI evidence from a valence decision task. Human Brain Mapping, 18, 30-41.

Manning, J. R., Polyn, S. M., Baltuch, G., Litt, B., \& Kahana, M. J. (2011). Oscillatory patterns in temporal lobe reveal context reinstatement during memory search. Proceedings of the National Academy of Sciences, U.S.A., 108, 12893-12897.

Manning, J. R., Sperling, M. R., Sharan, A., Rosenberg, E. A., \& Kahana, M. J. (2012). Spontaneously reactivated patterns in frontal and temporal lobe predict semantic clustering during memory search. Journal of Neuroscience, 32, 8871-8878.

Montoya, C., \& Sainsbury, R. (1985). The effects of entorhinal cortex lesions on type 1 and type 2 theta. Physiology $\varepsilon$ Behavior, 35, 121-126.

Palva, J. M., Palva, S., \& Kaila, K. (2005). Phase synchrony among neuronal oscillations in the human cortex. Journal of Neuroscience, 25, 3962-3972.
Polyn, S. M., \& Sederberg, P. B. (2014). Brain rhythms in mental time travel. Neuroimage, 85, 678-684.

Rugg, M. D., \& Vilberg, K. L. (2013). Brain networks underlying episodic memory retrieval. Current Opinion in Neurobiology, 23, 255-260.

Sederberg, P. B., Kahana, M. J., Howard, M. W., Donner, E. J., \& Madsen, J. R. (2003). Theta and gamma oscillations during encoding predict subsequent recall. Journal of Neuroscience, 23, 10809-10814.

Sederberg, P. B., Schulze-Bonhage, A., Madsen, J. R., Bromfield, E. B., Litt, B., Brandt, A., et al. (2007). Gamma oscillations distinguish true from false memories. Psychological Science, 18, 927-932.

Smith, A., Henson, R., Dolan, R., \& Rugg, M. (2004). fMRI correlates of the episodic retrieval of emotional contexts. Neuroimage, 22, 868-878.

Spaniol, J., Davidson, P. S., Kim, A. S., Han, H., Moscovitch, M., \& Grady, C. L. (2009). Event-related fMRI studies of episodic encoding and retrieval: Meta-analyses using activation likelihood estimation. Neuropsychologia, 47, 1765-1779.

Sugiura, M., Shah, N. J., Zilles, K., \& Fink, G. R. (2005). Cortical representations of personally familiar objects and places: Functional organization of the human posterior cingulate cortex. Journal of Cognitive Neuroscience, 17, 183-198.

Talk, A., Kang, E., \& Gabriel, M. (2004). Independent generation of theta rhythm in the hippocampus and posterior cingulate cortex. Brain Research, 1015, 15-24.

Thakral, P. P., Wang, T. H., \& Rugg, M. D. (2015). Cortical reinstatement and the confidence and accuracy of source memory. Neuroimage, 109, 118-129.

van Vugt, M. K., Sederberg, P. B., \& Kahana, M. J. (2007). Comparison of spectral analysis methods for characterizing brain oscillations. Journal of Neuroscience Methods, 162, 49-63.

Vilberg, K. L., \& Rugg, M. D. (2009). Functional significance of retrieval-related activity in lateral parietal cortex: Evidence from fMRI and ERPs. Human Brain Mapping, 30, 1490-1501.

Voytek, B., D'esposito, M., Crone, N., \& Knight, R. T. (2013). A method for event-related phase/amplitude coupling. Neuroimage, 64, 416-424.

Wagner, A. D., Shannon, B. J., Kahn, I., \& Buckner, R. L. (2005). Parietal lobe contributions to episodic memory retrieval. Trends in Cognitive Sciences, 9, 445-453.

Watrous, A. J., Fried, I., \& Ekstrom, A. D. (2011). Behavioral correlates of human hippocampal delta and theta oscillations during navigation. Journal of Neurophysiology, 105, 1747-1755.

Watrous, A. J., Lee, D. J., Izadi, A., Gurkoff, G. G., Shahlaie, K., \& Ekstrom, A. D. (2013). A comparative study of human and rat hippocampal low-frequency oscillations during spatial navigation. Hippocampus, 23, 656-661.

Watrous, A. J., Tandon, N., Conner, C. R., Pieters, T., \& Ekstrom, A. D. (2013). Frequency-specific network connectivity increases underlie accurate spatiotemporal memory retrieval. Nature Neuroscience, 16, 349-356. 